\title{
Resonances and $O$-curves in Hamiltonian systems
}

\author{
Paolo Buttà* $\quad$ Piero Negrini*
}

November 15, 2018

\begin{abstract}
We investigate the problem of the existence of trajectories asymptotic to elliptic equilibria of Hamiltonian systems in the presence of resonances.
\end{abstract}

\section{Introduction}

In the recent Congress held in Saint Petersbourg, dedicated to the $50^{\text {th }}$ anniversary of A.M. Lyapunov, the V.V. Kozlov conference [4] has been devoted to the so-called Lyapunov's First Method, applied in particular to the problem of the existence of $\mathrm{O}^{+}$-curves (resp. $\mathrm{O}^{-}$-curves) that are integral curves asymptotic in the future (resp. in the past) to the equilibria of Lagrangian systems. This problem is obviously related to the problem of the inversion of Dirichlet-Lagrange Theorem and the papers [5,9] contain the first important results for analytic potential functions with degenerate critical point. We refer to [8] for a review on further researches in this subject.

In the framework of Hamiltonian systems, a large number of papers have been devoted to the study of the relationship between instability (as well stability) of equilibria and resonances. We quote here just some essential references $[2,3,7,11]$ : in all these papers the instability was proved by constructing suitable Cetaev's functions.

We were therefore stimulated to study, by means of the First Method, the existence of $O$-curves of Hamiltonian systems in the presence of resonances. The Hamiltonian function we consider is supposed to have a non degenerate elliptic fixed point $F$ (see Section 2). The corresponding Birkhoff normal form turns out to be a perturbation of an integrable Hamiltonian function. Moreover the integrable system admits two distinct straight lines $r_{ \pm}$which are $O^{ \pm}$-curves. Following the russian terminology, these orbits are called "rays of the model system". Their existence is the starting point to build up the Cetaev function for the complete Hamiltonian system (resonances of order 3 or 4 are considered in [3]). In Section 3 we prove that the complete Hamiltonian system admits

\footnotetext{
*Dipartimento di Matematica, Università di Roma "La Sapienza", P.le Aldo Moro 2, 00185 Roma, Italy. E-mail: butta@mat.uniroma1.it, piero.negrini@uniroma1.it. Fax: +39-0644701007.
} 
$O$-curves, that have as asymptotic direction one of the two rays of the model system.

To prove this result we analyze the Hamiltonian system in a neighborhood of each ray. Then, by using a suitable set of variables we build up a new differential system with a non degenerate hyperbolic equilibrium. According to the choice of the ray, the relationship between these two differential systems allows us to conclude that the local stable manifold of this hyperbolic equilibrium corresponds either to a family of $\mathrm{O}^{+}$-curves or to a family of $\mathrm{O}^{-}$-curves of the Hamiltonian system.

Before concluding, let us recall that the existence of $O$-curves was analyzed by Lyapunov in the case of analytic differential systems. Many results on this subject can be found in the book of Zubov [12]. In particular, in Chapter III, perturbations of homogeneous polynomial differential systems having rays are studied and $O$-curves are obtained as power series of the variable $t^{-\alpha}, \alpha$ being a positive rational number depending on the degree of the homogeneous system. Moreover, in the general case, the coefficient of each term in the expansion is found to be a polynomial function of the variable $\log t$.

The Hamiltonian functions we consider here are just $C^{r}$ functions (the integer $r$ depending on the degree of the resonance) therefore we need to apply general hyperbolic theory, i.e. fixed point theorems in suitable functional spaces.

In the case $C^{\infty}$ the method of expansion in power series could still be used, at least at a formal level. In fact the existence of formal series representing $O$-curves is effective: a deep result by Kutsnesov [6] allows to conclude that there exist true $O$-curves having the formal series as asymptotic expansion.

In conclusion, we can repeat here the words one can read at the end of the introduction to Chapter III of the book of Zubov : "the basic ideas of almost all the results are in the works of Lyapunov".

\section{Preliminaries}

We start by considering a Hamiltonian function $H \in C^{\infty}(\Omega, \mathbb{R}), \Omega$ a domain in $\mathbb{R}^{2 n}$, containing the critical point $x=0$. Precisely, we assume that $x=0$ is an elliptic critical point, and therefore we write

$$
H(x)=H_{[2]}(x)+\mathcal{H}(x),
$$

where

$$
H_{[2]}(x)=\sum_{i=1}^{n} \omega_{i}\left(x_{i}^{2}+x_{i+n}^{2}\right), \quad \mathcal{H}(x)=O\left(|x|^{3}\right) .
$$

We consider the case in which the quadratic form $H_{[2]}$ has non trivial zeros. Moreover we assume the following hypotheses.

$\left(H_{1}\right)$ There exists an integer $N \geq 3$ such that, for any $h=\left(h_{1}, \ldots, h_{n}\right) \in$ $\mathbb{Z}_{+}^{n} \backslash\{0\},|h|:=\sum_{i=1}^{n} h_{i} \leq N-1$,

$$
\langle\omega, h\rangle \neq 0 .
$$


$\left(H_{2}\right)$ There exists a vector $k, k \in \mathbb{Z}_{+}^{n} \backslash\{0\},|k|=N$, such that

$$
\langle\omega, k\rangle=0 .
$$

Moreover, if $k^{\prime} \in \mathbb{Z}_{+}^{n} \backslash\{0, k\}$ is such that $\left\langle\omega, k^{\prime}\right\rangle=0$ then $\left|k^{\prime}\right|>M:=$ $3 N-1$.

In other words we assume a unique direction of resonance along $e:=\frac{k}{|k|}$, up to order $3 N$.

Under the previous hypotheses, by means of a symplectic change of variables $x=y+\Phi(y)$ in a neighborhood of $x=0$, we obtain the Birkhoff form of the Hamiltonian [10],

$$
H^{B}(I, \theta)=\sum_{j=1}^{\frac{3}{2} N} H_{[j]}^{B}(I)+H_{R}^{B}(I,\langle\theta, k\rangle)+\mathcal{R}^{B}(I, \theta),
$$

where we also introduced the action-angle variables $I=\left(I_{1}, \ldots, I_{n}\right), \theta=$ $\left(\theta_{1}, \ldots, \theta_{n}\right)$, with

$$
\left\{\begin{array}{l}
y_{i}=\sqrt{2 I_{i}} \cos \theta_{i} \\
y_{i+n}=\sqrt{2 I_{i}} \sin \theta_{i}
\end{array} \quad i=1, \ldots, n\right.
$$

In (2.1) we have:

- $H_{[j]}^{B}(I), j \in\left\{1, \ldots, \frac{3}{2} N\right\}$, is a homogeneous polynomial of degree $j$ in the variables $\left(I_{1}, \ldots, I_{n}\right)$. In particular

$$
H_{[1]}^{B}=\langle\omega, I\rangle .
$$

- $H_{R}^{B}$, the resonant term, takes the form

$$
H_{R}^{B}(I,\langle\theta, k\rangle)=\sum_{r=0}^{N} H_{r}(I,\langle\theta, k\rangle),
$$

where, for $r \leq N-1$,

$$
H_{r}=\sum_{j_{1}+\ldots+j_{n}=r}\left\{h_{j_{1}, \ldots, j_{n}}^{(1, r)} \cos \langle\theta, k\rangle+h_{j_{1}, \ldots, j_{n}}^{(2, r)} \sin \langle\theta, k\rangle\right\} \prod_{\alpha=1}^{n} I_{\alpha}^{\frac{k_{\alpha}}{2}+j_{\alpha}}
$$

and

$$
\begin{aligned}
H_{N}= & \sum_{j_{1}+\ldots+j_{n}=N}\left\{h_{j_{1}, \ldots, j_{n}}^{(1, N)} \cos \langle\theta, k\rangle+h_{j_{1}, \ldots, j_{n}}^{(2, N)} \sin \langle\theta, k\rangle\right. \\
& \left.+h_{j_{1}, \ldots, j_{n}}^{(3, N)} \cos 2\langle\theta, k\rangle+h_{j_{1}, \ldots, j_{n}}^{(4, N)} \sin 2\langle\theta, k\rangle\right\} \prod_{\alpha=1}^{n} I_{\alpha}^{\frac{k_{\alpha}}{2}+j_{\alpha}} .
\end{aligned}
$$


- $\mathcal{R}^{B}(I, \theta)$ collects the higher order terms:

$$
\mathcal{R}^{B}(I, \theta)=O\left(|I|^{\frac{3 N+1}{2}}\right) .
$$

We now make two further assumptions on the system.

$\left(H_{3}\right) I=k$ is a channel of instability, that is

$$
H_{[j]}^{B}(k)=0 \quad \forall j \in\left\{1, \ldots, \frac{N-1}{2}\right\} .
$$

$\left(H_{4}\right)$ Let $\Psi: \mathbb{R} \rightarrow \mathbb{R}$ be defined by setting

$$
\Psi(\sigma):=H_{\left[\frac{1}{2} N\right]}^{B}(k)+H_{0}\left(k, k_{1}^{2} \sigma\right), \quad \sigma \in \mathbb{R},
$$

with $H_{\left[\frac{1}{2} N\right]}^{B}$ and $H_{0}$ as in (2.1) and (2.2) respectively. We assume that $\Psi$ has a simple zero at $\sigma=c$.

Remark 2.1 The assumption $\left(H_{3}\right)$ is satisfied if $N \leq 4$, otherwise it implies that Arnol'd condition of non degeneracy [1] is violated up to the order $N$.

Remark 2.2 The general form of (2.3) can be written

$$
\Psi(\sigma)=A \cos \left[k_{1}^{2}\left(\sigma+\sigma_{0}\right)\right]+B .
$$

The hypothesis $\left(H_{4}\right)$ implies that there exist two roots $c_{ \pm}$of $\Psi$ such that $\Psi^{\prime}\left(c_{+}\right) \Psi^{\prime}\left(c_{-}\right)<0$. Let $\Psi^{\prime}\left(c_{+}\right)>0$. Then the ray $r_{+}$(resp. $r_{-}$) corresponds to $c_{+}$(resp. $c_{-}$). Obviously, if $N$ is odd then $B=0$ and therefore $\Psi^{\prime \prime}\left(c_{ \pm}\right)=$ $-k_{1}^{4} \Psi\left(c_{ \pm}\right)=0$. This fact will be exploited in Section 3 .

We are now able to state the main result of the paper.

Theorem 2.3 Under Hypotheses $\left(H_{1}\right),\left(H_{2}\right),\left(H_{3}\right)$, and $\left(H_{4}\right)$ the Hamiltonian system admits a $(n-1)$-parameters family of $\mathrm{O}^{+}$-curves as well as a $(n-1)$ parameters family of $\mathrm{O}^{-}$-curves.

The proof of the theorem is given in the next section.

Remark 2.4 The theorem still hold if we assume $H \in C^{r}(\Omega, \mathbb{R}), r=3 N+1$.

\section{Proof of Theorem 2.3}

Without loss of generality we may assume $k_{1}>0$. We then introduce the symplectic linear change of coordinates $(I, \theta) \rightarrow(J, \psi)=\left(A_{1} I, A_{2} \theta\right)$ given by

$$
\begin{array}{ccc}
J_{1}=k_{1} I_{1}, & J_{\alpha}=k_{1} I_{\alpha}-k_{\alpha} I_{1}, & \alpha=2, \cdots, n, \\
\psi_{1}=\frac{\langle\theta, k\rangle}{k_{1}^{2}}, & \psi_{\alpha}=\frac{\theta_{\alpha}}{k_{1}}, & \alpha=2, \cdots, n .
\end{array}
$$


The Hamiltonian function $K(J, \psi)=H\left(A_{1}^{-1} J, A_{2}^{-1} \psi\right)$ then reads

$$
K(J, \psi)=K(J)+K^{R}\left(J, \psi_{1}\right)+\mathcal{R}(J, \psi),
$$

where

$$
\begin{aligned}
& K(J)=\sum_{j=1}^{\frac{3}{2} N} K_{[j]}(J), \quad K_{[j]}(J)=H_{[j]}^{B}\left(A_{1}^{-1} J\right), \\
& K^{R}\left(J, \psi_{1}\right)=\sum_{r=0}^{N} K_{r}\left(J, \psi_{1}\right), \quad K_{r}\left(J, \psi_{1}\right)=H_{r}\left(A_{1}^{-1} J, k_{1}^{2} \psi_{1}\right), \\
& \mathcal{R}(J, \psi)=\mathcal{R}^{B}\left(A_{1}^{-1} J, A_{2}^{-1} \psi\right) .
\end{aligned}
$$

We next introduce the following notation for $n$-components vectors,

$$
x=\left(x_{1}, \hat{x}\right), \quad \hat{x} \equiv\left(x_{2}, \ldots, x_{n}\right) .
$$

The Hamiltonian system then reads

$$
\left\{\begin{array}{l}
\dot{J}_{1}=-\frac{\partial K^{R}\left(J_{1}, \hat{J}, \psi_{1}\right)}{\partial \psi_{1}}-\frac{\partial \mathcal{R}\left(J_{1}, \hat{J}, \psi_{1}, \hat{\psi}\right)}{\partial \psi_{1}}, \\
\dot{\hat{J}}=-\frac{\partial \mathcal{R}\left(J_{1}, \hat{J}, \psi_{1}, \hat{\psi}\right)}{\partial \hat{\psi}}, \\
\dot{\psi}_{1}=\Omega_{1}\left(J_{1}, \hat{J}\right)+\frac{\partial K^{R}\left(J_{1}, \hat{J}, \psi_{1}\right)}{\partial J_{1}}+\frac{\partial \mathcal{R}\left(J_{1}, \hat{J}, \psi_{1}, \hat{\psi}\right)}{\partial J_{1}} \\
\dot{\hat{\psi}}=\hat{\Omega}\left(J_{1}, \hat{J}\right)+\frac{\partial K^{R}\left(J_{1}, \hat{J}, \psi_{1}\right)}{\partial \hat{J}}+\frac{\partial \mathcal{R}\left(J_{1}, \hat{J}, \psi_{1}, \hat{\psi}\right)}{\partial \hat{J}}
\end{array}\right.
$$

where

$$
\Omega_{1}(J)=\frac{\partial K(J)}{\partial J_{1}}, \quad \hat{\Omega}(J)=\frac{\partial K(J)}{\partial \hat{J}} .
$$

We observe that, by virtue of Hypotheses $\left(H_{3}\right)$ and $\left(H_{4}\right)$, since $A_{1}^{-1}\left(J_{1}, 0\right)=$ $J_{1} k_{1}^{-2} k$,

$$
\Omega_{1}\left(J_{1}, 0\right)+\frac{\partial K^{R}\left(J_{1}, 0, c\right)}{\partial J_{1}}=\sum_{j=\frac{N}{2}+\delta}^{\frac{3}{2} N} \frac{\partial K_{[j]}}{\partial J_{1}}\left(J_{1}, 0\right)+\sum_{r=1}^{N} \frac{\partial K_{r}\left(J_{1}, 0, c\right)}{\partial J_{1}}
$$

where

$$
\delta= \begin{cases}1 / 2 & \text { if } N \text { is odd } \\ 1 & \text { if } N \text { is even }\end{cases}
$$

and

$$
\gamma:=\frac{\partial K_{0}}{\partial \psi_{1}}(1,0, c) \neq 0
$$


For definiteness we consider the case $\gamma>0$; the case $\gamma<0$ will be briefly discussed at the end of the section. We look for solutions to (3.2) of the form

$$
\begin{aligned}
J_{1}(t) & =\left[\frac{2}{(N-2) t}\right]^{\frac{2}{N-2}}\left\{\Gamma+u_{1}(t)\right\}, \\
\hat{J}(t) & =\left[\frac{2}{(N-2) t}\right]^{\frac{N+2}{N-2}} \hat{u}(t), \\
\psi_{1}(t) & =c+\left[\frac{2}{(N-2) t}\right]^{\frac{2 \delta}{N-2}}\left\{c_{1}+c_{2}\left[\frac{2}{(N-2) t}\right]^{\frac{2 \delta}{N-2}}+v_{1}(t)\right\}, \\
\hat{\psi}(t) & =\left[\frac{2}{(N-2) t}\right]^{-1}\left\{\hat{\Omega}_{0}+\hat{v}(t)\right\},
\end{aligned}
$$

where

$$
\Gamma:=\gamma^{-\frac{2}{N-2}}, \quad \hat{\Omega}_{0}:=\frac{2}{k_{1}(N-2)}\left(\omega_{2}, \ldots, \omega_{n}\right)
$$

and $u(t)=\left(u_{1}(t), \hat{u}(t)\right), v(t)=\left(v_{1}(t), \hat{v}(t)\right)$ are such that

$$
\lim _{t \rightarrow \infty} u(t)=0, \quad \lim _{t \rightarrow \infty} v(t)=0 .
$$

The explicit values of the parameters $c_{1}$ and $c_{2}$ are given in Proposition 3.1 below. We introduce the new independent variable $z$ by setting

$$
z=\left[\frac{2}{(N-2) t}\right]^{\frac{2}{N-2}}
$$

and write the differential equations for the functions

$$
\xi(z):=u(t(z))), \quad \eta(z):=v(t(z)) .
$$

This is the content of the following proposition.

Proposition 3.1 Let, for $N$ odd,

$$
c_{1}=-\frac{2 \Gamma^{\frac{N-1}{2}}}{N+1} \frac{\partial K_{\left[\frac{N+1}{2}\right]}(1,0)}{\partial J_{1}}, \quad c_{2}=-\frac{2 \Gamma^{\frac{N}{2}}}{N+2} \frac{\partial K_{1}(1,0, c)}{\partial J_{1}}
$$

and, for $N$ even,

$$
c_{1}=-\frac{2 \Gamma^{\frac{N}{2}}}{N+2}\left\{\frac{\partial K_{\left[\frac{N}{2}+1\right]}(1,0)}{\partial J_{1}}+\frac{\partial K_{1}(1,0, c)}{\partial J_{1}}\right\}, \quad c_{2}=0
$$


The functions $(\xi(z), \eta(z))$ are solutions to the differential system

$$
\left\{\begin{array}{l}
z \frac{d \xi_{1}}{d z}=\frac{N-2}{2} \xi_{1}+d_{0} z+U_{1}(z, \xi, \eta), \\
z \frac{d \hat{\xi}}{d z}=-\frac{N+2}{2} \hat{\xi}+\hat{U}(z, \xi, \eta), \\
z \frac{d \eta_{1}}{d z}=-\frac{N+2 \delta}{2} \eta_{1}+d_{1} z+d_{2} \xi_{1}+V_{1}(z, \xi, \eta), \\
z \frac{d \hat{\eta}}{d z}=\frac{N-2}{2} \hat{\eta}+\hat{d} z+\hat{V}(z, \xi, \eta),
\end{array}\right.
$$

where, for $N$ odd,

$$
\begin{aligned}
& d_{0}=\Gamma^{\frac{N+2}{2}} \frac{\partial K_{1}(1,0, c)}{\partial \psi_{1}}-\Gamma \frac{k_{1}^{4}}{2} c_{1}^{2}, \\
& d_{1}=-\Gamma^{\frac{N+1}{2}} \frac{\partial K_{\left[\frac{N+3}{2}\right]}(1,0)}{\partial J_{1}}-c_{1} \Gamma^{\frac{N}{2}} \frac{\partial^{2} K_{1}(1,0, c)}{\partial J_{1} \partial \psi_{1}} \\
& d_{2}=-\frac{N-1}{2} \Gamma^{\frac{N-3}{2}} \frac{\partial K_{\left[\frac{N+1}{2}\right]}(1,0)}{\partial J_{1}}-c_{1} \Gamma^{-1} \frac{N(N-2)}{4}
\end{aligned}
$$

and, for $N$ even,

$$
\begin{aligned}
d_{0}= & \Gamma^{\frac{N+2}{2}} \frac{\partial K_{1}(1,0, c)}{\partial \psi_{1}}+c_{1} \frac{\partial^{2} K_{0}(1,0, c)}{\partial \psi_{1}^{2}} \\
d_{1}= & -\Gamma^{\frac{N}{2}+1}\left\{\frac{\partial K_{\left[\frac{N}{2}+2\right]}(1,0)}{\partial J_{1}}+\frac{\partial K_{2}(1,0, c)}{\partial J_{1}}\right\}-c_{1} \Gamma^{\frac{N}{2}} \frac{\partial^{2} K_{1}(1,0, c)}{\partial J_{1} \partial \psi_{1}} \\
& +k_{1}^{4} c_{1}^{2} \Gamma^{\frac{N-2}{2}} \frac{\partial K_{0}(1,0, c)}{\partial J_{1}}, \\
d_{2}= & -\frac{N}{2} \Gamma^{\frac{N}{2}-1} \frac{\partial K_{\left[\frac{N}{2}+1\right]}(1,0)}{\partial J_{1}}-c_{1} \Gamma^{-1} \frac{N(N-2)}{4} .
\end{aligned}
$$

Finally, for any $N$,

$$
\hat{d}=\Gamma^{2}\left\{\frac{\partial K_{[2]}(1,0)}{\partial \hat{J}}+\delta_{N, 4} \frac{\partial K_{0}(1,0, c)}{\partial \hat{J}}\right\} .
$$

In particular $\hat{d}=0$ if $N=3$.

The functions $U=\left(U_{1}, \hat{U}\right)$ and $V=\left(V_{1}, \hat{V}\right)$ are $C^{1}$ functions in a "right neighborhood" $\mathcal{N}^{+}$of zero in $\mathbb{R}^{2 n+1}$,

$$
\mathcal{N}^{+}:=\left\{(z, \xi, \eta) \in \mathbb{R}^{2 n+1}: z \in[0, \varepsilon),\|(\xi, \eta)\|<\varepsilon\right\} .
$$

Moreover the Jacobian matrices satisfy

$$
D U(0,0,0)=0, \quad D V(0,0,0)=0 .
$$


Proof. The proof is straightforward but cumbersome. Of course one has to rewrite the system (3.2) in terms of the independent variable $z$ and the unknowns $(\xi, \eta)$, according to (3.6). The main point is then to extract from the r.h.s. of the system the linear part and verify the regularity property of the remainder.

By (3.5) and (3.6) we have

$$
\begin{aligned}
& J_{1}=z\left(\Gamma+\xi_{1}\right), \quad \hat{J}=z^{\frac{N+2}{2}} \hat{\xi}, \quad \psi_{1}=c+z^{\delta}\left(c_{1}+c_{2} z^{\delta}+\eta_{1}\right), \\
& \hat{\psi}=z^{-\frac{N-2}{2}}\left(\hat{\Omega}_{0}+\hat{\eta}\right),
\end{aligned}
$$

whence, since $\dot{z}=-z^{\frac{N}{2}}$,

$$
\left\{\begin{array}{l}
-z^{-\frac{N}{2}} \dot{J}_{1}=\Gamma+\xi_{1}+z \frac{d \xi_{1}}{d z} \\
-z^{-\frac{N}{2}} \dot{\hat{J}}=\frac{N+2}{2} z^{\frac{N}{2}} \hat{\xi}+z^{\frac{N+2}{2}} \frac{d \hat{\xi}}{d z} \\
-z^{-\frac{N}{2}} \dot{\psi}_{1}=c_{1} \delta z^{\delta-1}+2 c_{2} \delta z^{2 \delta-1}+\delta z^{\delta-1} \eta_{1}+z^{\delta} \frac{d \eta_{1}}{d z} \\
-\dot{\hat{\psi}}=-\frac{N-2}{2}\left(\hat{\Omega}_{0}+\hat{\eta}\right)+z \frac{d \hat{\eta}}{d z}
\end{array}\right.
$$

By comparing (3.9) and (3.14) it follows that

$$
\begin{aligned}
& U_{1}(z, \xi, \eta)=-z^{-\frac{N}{2}} \dot{J}_{1}-\Gamma-\frac{N}{2} \xi_{1}-d_{0} z, \quad \hat{U}(z, \xi, \eta)=-z^{-N} \dot{\hat{J}} \\
& V_{1}(z, \xi, \eta)=-z^{1-\delta-\frac{N}{2}} \dot{\psi}_{1}-c_{1} \delta-2 c_{2} \delta z^{\delta}+\frac{N}{2} \eta_{1}-d_{1} z-d_{2} \xi_{1} \\
& \hat{V}(z, \xi, \eta)=-\dot{\hat{\psi}}+\frac{N-2}{2} \hat{\Omega}_{0}-\hat{d} z
\end{aligned}
$$

where the time derivatives $(\dot{J}, \dot{\psi})$ are given by the r.h.s. of (3.2) expressed in terms of the variables $(z, \xi, \eta)$ by means of (3.13).

In the sequel we shall denote by $\mathcal{N L T}$ a generic $C^{1}$ function of $(z, \xi, \eta)$ which vanishes with its first partial derivatives in $(0,0,0)$. Since

$$
A_{1}^{-1} J=\frac{1}{k_{1}} J+\frac{J_{1}}{k_{1}^{2}}(0, \hat{k})=z\left\{\frac{\Gamma+\xi_{1}}{k_{1}^{2}}\left(k_{1}, \hat{k}\right)+z^{\frac{N}{2}} \frac{1}{k_{1}}(0, \hat{\xi})\right\},
$$

recalling the definition of $\mathcal{R}$ in (3.1) we have:

$$
\begin{aligned}
& \frac{\partial \mathcal{R}\left(z\left(\Gamma+\xi_{1}\right), z^{\frac{N+2}{2}} \hat{\xi}, c+\sqrt{z}\left(c_{1}+c_{2} \sqrt{z}+\eta_{1}\right), z^{-\frac{N-2}{2}}\left(\hat{\Omega}_{0}+\hat{\eta}\right)\right)}{\partial \psi}=z^{N} \mathcal{N} \mathcal{L}, \\
& \frac{\partial \mathcal{R}\left(z\left(\Gamma+\xi_{1}\right), z^{\frac{N+2}{2}} \hat{\xi}, c+\sqrt{z}\left(c_{1}+c_{2} \sqrt{z}+\eta_{1}\right), z^{-\frac{N-2}{2}}\left(\hat{\Omega}_{0}+\hat{\eta}\right)\right)}{\partial J}=z^{\frac{N}{2}} \mathcal{N} \mathcal{L T} .
\end{aligned}
$$


By the second equality in (3.15) and (3.19) we get $\hat{U}=\mathcal{N L T}$. Moreover, by (3.17), (3.20) and the definition (3.12) of $\hat{d}$ it is straightforward to conclude also that $\hat{V}=\mathcal{N L T}$, we omit the details. The analysis of the functions $U_{1}$ and $V_{1}$ is more delicate and the cases $N$ odd and $N$ even have to be treated separately.

Case $N$ odd. By Remark 2.2 we have

$$
\frac{\partial^{2} K_{0}(1,0, c)}{\partial \psi_{1}^{2}}=0, \quad \frac{\partial^{3} K_{0}(1,0, c)}{\partial \psi_{1}^{3}}=-k_{1}^{4} \frac{\partial K_{0}(1,0, c)}{\partial \psi_{1}} .
$$

By the definitions (3.1), (3.18), and recalling $\delta=\frac{1}{2}$ in this case,

$$
\begin{aligned}
\left.\frac{\partial K_{0}\left(z\left(\Gamma+\xi_{1}\right), z^{\frac{N+2}{2}} \hat{\xi}, c\right.}{\partial \psi_{1}} \sqrt{z}\left(c_{1}+c_{2} \sqrt{z}+\eta_{1}\right)\right) \\
=z^{\frac{N}{2}} \frac{\partial K_{0}\left(\Gamma+\xi_{1}, z^{\frac{N}{2}} \hat{\xi}, c+\sqrt{z}\left(c_{1}+c_{2} \sqrt{z}+\eta_{1}\right)\right)}{\partial \psi_{1}} \\
=z^{\frac{N}{2}}\left(\Gamma+\xi_{1}\right)^{\frac{N}{2}} \frac{\partial K_{0}(1,0, c)}{\partial \psi_{1}}\left\{1-\frac{k_{1}^{4}}{2} c_{1}^{2} z+\mathcal{N L T}\right\},
\end{aligned}
$$

while, for $r \geq 1$,

$$
\begin{aligned}
\left.\frac{\partial K_{r}\left(z\left(\Gamma+\xi_{1}\right), z^{\frac{N+2}{2}} \hat{\xi}, c\right.}{\partial}+\sqrt{z}\left(c_{1}+c_{2} \sqrt{z}+\eta_{1}\right)\right) \\
=z_{1} \\
=z^{\frac{N}{2}+r} \frac{\partial K_{r}\left(\Gamma+\xi_{1}, z^{\frac{N}{2}} \hat{\xi}, c+\sqrt{z}\left(c_{1}+c_{2} \sqrt{z}+\eta_{1}\right)\right)}{\partial \psi_{1}} \\
\left.\partial \xi_{1}\right)^{\frac{N}{2}+r} \frac{\partial K_{r}(1,0, c)}{\partial \psi_{1}}+z^{\frac{N}{2}} \mathcal{N L T} .
\end{aligned}
$$

Then, recalling the definitions (3.4), (3.10), and $\Gamma=\gamma^{-\frac{2}{N-2}}$,

$$
\begin{aligned}
-z^{-\frac{N}{2}} \dot{J}_{1} & =\gamma\left(\Gamma+\xi_{1}\right)^{\frac{N}{2}}\left(1-\frac{k_{1}^{4}}{2} c_{1}^{2} z\right)+\frac{\partial K_{1}(1,0, c)}{\partial \psi_{1}} \Gamma^{\frac{N+2}{2}} z+\mathcal{N L T} \\
& =\Gamma+\frac{N}{2} \xi_{1}+d_{0} z+\mathcal{N L T} .
\end{aligned}
$$

The previous expansions imply $U_{1}=\mathcal{N L} \mathcal{L}$.

Analogously, recalling also (3.3),

$$
\begin{aligned}
\dot{\psi}_{1}= & z^{\frac{N-1}{2}}\left\{\frac{\partial K_{\left[\frac{N+1}{2}\right]}\left(\Gamma+\xi_{1}, 0\right)}{\partial J_{1}}+\left(c_{1}+c_{2} \sqrt{z}+\eta_{1}\right) \frac{\partial^{2} K_{0}\left(\Gamma+\xi_{1}, 0, c\right)}{\partial J_{1} \partial \psi_{1}}\right\} \\
& +z^{\frac{N}{2}} \frac{\partial K_{1}\left(\Gamma+\xi_{1}, 0, c\right)}{\partial J_{1}}+c_{1}^{2} z^{\frac{N+1}{2}} \frac{\partial^{3} K_{0}\left(\Gamma+\xi_{1}, 0, c\right)}{\partial J_{1} \partial \psi_{1}^{2}} \\
& +c_{1} z^{\frac{N+1}{2}} \frac{\partial^{2} K_{1}\left(\Gamma+\xi_{1}, 0, c\right)}{\partial J_{1} \partial \psi_{1}}+z^{\frac{N+1}{2}} \frac{\partial K_{\left[\frac{N+3}{2}\right]}\left(\Gamma+\xi_{1}, 0\right)}{\partial J_{1}} \\
& +z^{\frac{N-1}{2}} \mathcal{N} \mathcal{L T}
\end{aligned}
$$


whence

$$
\begin{aligned}
z^{\frac{1-N}{2}} \dot{\psi}_{1}= & \left(\Gamma+\xi_{1}\right)^{\frac{N-1}{2}} \frac{\partial K_{\left[\frac{N+1}{2}\right]}(1,0)}{\partial J_{1}}+c_{1}\left(\Gamma+\xi_{1}\right)^{\frac{N-2}{2}} \frac{\partial^{2} K_{0}(1,0, c)}{\partial J_{1} \partial \psi_{1}} \\
& +\left(c_{2} \sqrt{z}+\eta_{1}\right)\left(\Gamma+\xi_{1}\right)^{\frac{N-2}{2}} \frac{\partial^{2} K_{0}(1,0, c)}{\partial J_{1} \partial \psi_{1}} \\
& +\sqrt{z} \Gamma^{\frac{N}{2}} \frac{\partial K_{1}(1,0, c)}{\partial J_{1}}+c_{1}^{2} z \Gamma^{\frac{N-2}{2}} \frac{\partial^{3} K_{0}(1,0, c)}{\partial J_{1} \partial \psi_{1}^{2}} \\
& +c_{1} z \Gamma^{\frac{N}{2}} \frac{\partial^{2} K_{1}(1,0, c)}{\partial J_{1} \partial \psi_{1}}+z \Gamma^{\frac{N+1}{2}} \frac{\partial K_{\left[\frac{N+3}{2}\right]}(1,0)}{\partial J_{1}} \\
& +z^{\frac{N-1}{2}} \mathcal{N L T} .
\end{aligned}
$$

On the other hand, by the explicit form of the functions $K_{0}$, recalling $|k|=N$ and Remark 2.2, it is easy to verify that

$$
\frac{\partial^{2} K_{0}(1,0, c)}{\partial J_{1} \partial \psi_{1}}=\frac{N}{2} \frac{\partial K_{0}(1,0, c)}{\partial \psi_{1}}=\frac{N}{2} \gamma, \quad \frac{\partial^{3} K_{0}(1,0, c)}{\partial J_{1} \partial \psi_{1}^{2}}=-k_{1}^{4} \frac{\partial K_{0}(1,0, c)}{\partial J_{1}}=0 .
$$

By inserting the previous expression of $z^{\frac{1-N}{2}} \dot{\psi}_{1}$ in (3.16), expanding up to the first order in the variable $\xi_{1}$, and recalling the definitions (3.7), (3.10) of $c_{1}, c_{2}$, $d_{1}$, and $d_{2}$, we get $V_{1}=\mathcal{N L T}$.

Case $N$ even. To prove $U_{1}=\mathcal{N L T}$ we argue as before; the only difference is that $\delta=1$ and $c_{2}=0$ in this case, so that

$$
\begin{aligned}
\frac{\partial K_{0}\left(z\left(\Gamma+\xi_{1}\right), z^{\frac{N+2}{2}} \hat{\xi}, c+z\left(c_{1}+\eta_{1}\right)\right)}{\partial \psi_{1}} \\
=z^{\frac{N}{2}}\left(\Gamma+\xi_{1}\right)^{\frac{N}{2}}\left\{\frac{\partial K_{0}(1,0, c)}{\partial \psi_{1}}+\frac{\partial^{2} K_{0}(1,0, c)}{\partial \psi_{1}^{2}} c_{1} z+\mathcal{N} \mathcal{L T}\right\},
\end{aligned}
$$

whence the definition of $d_{0}$ in (3.11) for $N$ even.

We finally have

$$
\begin{aligned}
\dot{\psi}_{1}= & z^{\frac{N}{2}}\left\{\frac{\partial K_{\left[\frac{N}{2}+1\right]}\left(\Gamma+\xi_{1}, 0\right)}{\partial J_{1}}+\left(c_{1}+\eta_{1}\right) \frac{\partial^{2} K_{0}\left(\Gamma+\xi_{1}, 0, c\right)}{\partial J_{1} \partial \psi_{1}}\right\} \\
& +z^{\frac{N}{2}} \frac{\partial K_{1}\left(\Gamma+\xi_{1}, 0, c\right)}{\partial J_{1}}++c_{1}^{2} z^{\frac{N}{2}+1} \frac{\partial^{3} K_{0}\left(\Gamma+\xi_{1}, 0, c\right)}{\partial J_{1} \partial \psi_{1}^{2}} \\
& +c_{1} z^{\frac{N}{2}+1} \frac{\partial^{2} K_{1}\left(\Gamma+\xi_{1}, 0, c\right)}{\partial J_{1} \partial \psi_{1}}+z^{\frac{N}{2}+1} \frac{\partial K_{\left[\frac{N}{2}+2\right]}\left(\Gamma+\xi_{1}, 0\right)}{\partial J_{1}} \\
& +z^{\frac{N}{2}+1} \frac{\partial K_{2}\left(\Gamma+\xi_{1}, 0, c\right)}{\partial J_{1}}+z^{\frac{N}{2}} \mathcal{N} \mathcal{L} \mathcal{},
\end{aligned}
$$


whence

$$
\begin{aligned}
z^{-\frac{N}{2}} \dot{\psi}_{1}= & \left(\Gamma+\xi_{1}\right)^{\frac{N}{2}} \frac{\partial K_{\left[\frac{N}{2}+1\right]}(1,0)}{\partial J_{1}}+c_{1}\left(\Gamma+\xi_{1}\right)^{\frac{N-2}{2}} \frac{\partial^{2} K_{0}(1,0, c)}{\partial J_{1} \partial \psi_{1}} \\
& +\Gamma^{\frac{N}{2}} \frac{\partial K_{1}(1,0, c)}{\partial J_{1}}+\eta_{1}\left(\Gamma+\xi_{1}\right)^{\frac{N-2}{2}} \frac{\partial^{2} K_{0}(1,0, c)}{\partial J_{1} \partial \psi_{1}} \\
& +c_{1} z \Gamma^{\frac{N}{2}} \frac{\partial^{2} K_{1}(1,0, c)}{\partial J_{1} \partial \psi_{1}}+c_{1}^{2} z \Gamma^{\frac{N-2}{2}} \frac{\partial^{3} K_{0}(1,0, c)}{\partial J_{1} \partial \psi_{1}^{2}} \\
& +z \Gamma^{\frac{N}{2}+1} \frac{\partial K_{\left[\frac{N}{2}+2\right]}(1,0)}{\partial J_{1}}+z \Gamma^{\frac{N}{2}+1} \frac{\partial K_{2}(1,0, c)}{\partial J_{1}}+\mathcal{N L T} .
\end{aligned}
$$

By inserting the previous expression of $z^{-\frac{N}{2}} \dot{\psi}_{1}$ in (3.16), expanding up to the first order in the variable $\xi_{1}$, using that in this case we still have

$$
\frac{\partial^{2} K_{0}(1,0, c)}{\partial J_{1} \partial \psi_{1}}=\frac{N}{2} \frac{\partial K_{0}(1,0, c)}{\partial \psi_{1}}=\frac{N}{2} \gamma, \quad \frac{\partial^{3} K_{0}(1,0, c)}{\partial J_{1} \partial \psi_{1}^{2}}=-k_{1}^{4} \frac{\partial K_{0}(1,0, c)}{\partial J_{1}}
$$

and recalling the definitions (3.8) and (3.11) of $c_{1}, c_{2}, d_{1}$, and $d_{2}$, we get $V_{1}=$ $\mathcal{N L T}$.

System (3.14) is equivalent to the autonomous system:

$$
\left\{\begin{array}{l}
\frac{d z}{d \tau}=-z \\
\frac{d \xi_{1}}{d \tau}=-\frac{N-2}{2} \xi_{1}-d_{0} z-U_{1}(z, \xi, \eta) \\
\frac{d \hat{\xi}}{d \tau}=\frac{N+2}{2} \hat{\xi}-\hat{U}(z, \xi, \eta) \\
\frac{d \eta_{1}}{d \tau}=\frac{N+2 \delta}{2} \eta_{1}-d_{1} z-d_{2} \xi_{1}-V_{1}(z, \xi, \eta) \\
\frac{d \hat{\eta}}{d \tau}=-\frac{N-2}{2} \hat{\eta}-\hat{d} z-\hat{V}(z, \xi, \eta)
\end{array}\right.
$$

The origin $(z, \xi, \eta)=(0,0,0)$ is an hyperbolic equilibrium. In order to apply the hyperbolic theory we have to get rid of the fact that the system is defined in $\mathcal{N}^{+}$, and not in a full neighborhood of the origin. However the proof of the existence of the stable manifold can be easily adapted to this case. Then we have a local invariant stable manifold, parametrized by $\left(z, \xi_{1}, \hat{\eta}\right)$, which is the graph of a $C^{1}$ function defined in a neighborhood $B_{\varepsilon} \subset \mathcal{N}^{+}$.

In conclusion, coming back to the original variables we have obtained $W^{+}$, an $(n-1)$-dimensional surface of $\mathrm{O}^{+}$-curves.

We conclude this section by briefly considering the case $\gamma<0$. We consider the problem (3.2) in the past, or equivalently, we change the sign of the Hamiltonian function and let $t>0$. In particular we replace $\gamma$ by $|\gamma|$ everywhere it 
appears. We thus get again a system of the same form of (3.21). In conclusion, to the stable manifold corresponds now $W^{-}$, an $(n-1)$-dimensional surface of $\mathrm{O}^{-}$-curves.

Finally, recalling that we have two different rays of the model system, the proof of Theorem 2.3 is accomplished.

\section{References}

[1] V.I. Arnold. Small denominators and the problems of stability of motions in classical and celestial mechanics. Uspekhi Mat. Nauk (6). 1963. V. 18. P. 91-192.

[2] H.E. Cabral, K. Meyer. Stability of equilibria and fixed points of conservative systems. Nonlinearity. 1999. V. 12. P. 1351-1362.

[3] L.G. Khazin. On the stability of Hamiltonian systems in the presence of resonances. Prikl. Mat. Mekh. (3). 1971. V. 35. P. 423-431.

[4] V.V. Kozlov. First Lyapunov method for strongly nonlinear systems of differential equations. Congress (2007, June 4-8) in Saint Petersbourg, dedicated to the $150^{\text {th }}$ anniversary of A.M. Lyapunov.

[5] V.V. Kozlov. Asymptotic motions and the inversion of Lagrange-Dirichlet Theorem. Prikl. Mat. Mekh. (4). 1986. V. 27. P. 719-725.

[6] A.N. Kuznetsov. Differentiable solutions to degenerate systems of ordinary equations. Functional Analysis and its Applications. 1972. V. 6. P. 119-127.

[7] A.P. Markeev. The problem of the stability of the equilibrium position of a Hamiltonian system at 3:1 resonance. J. Appl. Maths. and Mech. 2001. V. 65. P. 639-645.

[8] P. Negrini. On the inversion of Lagrange-Dirichlet theorem. Resenhas IMEUSP. 1995. V. 2 (1). P. 83-114.

[9] V.V. Kozlov, V. Palamadov. On asymptotic solutions of the equations of classical mechanics. Sov. Math. Dokl. 1982. V. 25. P. 286-289.

[10] C.L. Siegel, J.K. Moser. Lectures on Celestial Mechanics. Springer-Verlag. 1971.

[11] A.G. Sokol'skii. On the stability of autonomous Hamiltonian system with two degrees of freedom under first-order resonance. J. Appl. Maths. and Mech. 1977. V. 141. P. 24-33.

[12] V.I. Zubov. Methods of A.M. Lyapunov and Their Applications. Transl. by P. Noordhoff - LTD - Groningen - The Netherlands. 1964. 